\title{
Use of Point-of-Care Tests (POCTs) by US Primary Care Physicians
}

\author{
Augustine J. Sohn, MD, MPH, John M. Hickner, MD, MSc, and Fasika Alem, MPH
}

Objective: Point-of-care testing (POCT) has been used in the United States for several decades to diagnose and monitor acute and chronic medical conditions. The aim of this study is to assess the use of POCT and perceived benefits of and concerns regarding POCT among US family physicians.

Methods: A total of $\mathbf{4 0 5}$ US family physicians responded to an electronic survey about their use of POCT for diagnosing and monitoring illnesses and for reducing referrals for specialty care. Respondents were also asked about the frequency of, benefits of, and concerns regarding the use of POCT.

Results: The top 10 conditions for which physicians reported using POCT for diagnosis are diabetes mellitus, urinary tract infections, strep throat, influenza, pregnancy, anemia, infectious mononucleosis, anticoagulation, acute cardiac conditions, and lipid disorders. More than half of the respondents use or would use $>15$ kinds of POCTs at least weekly. The perceived benefits of POCT included immediately available results and physician/patient satisfaction; perceived concerns included the accuracy and cost of the tests.

Conclusions: Findings show that a variety of point-of-care tests are used by US family physicians for immediate diagnosis and monitoring. With continuing technical improvements and decreasing costs, it is highly likely that POCT use will increase dramatically. (J Am Board Fam Med 2016;29:371-376.)

Keywords: Physicians, Family; Point-of-Care Systems

Point-of-care testing (POCT), sometimes referred to as near-patient or bedside testing, is defined as testing done at the site of patient care with rapid turnaround of results that enables immediate diagnosis and treatment plans. ${ }^{1}$ POCT in physician offices has been used for decades in the United States to diagnose acute conditions such as pregnancy, urinary tract infection, and streptococcal pharyngitis. POCT has recently been used to diagnose and monitor chronic medical conditions such as diabetes mellitus ${ }^{2-5}$ and to monitor the international normalized ratio (INR) of patients receiving warfarin therapy. ${ }^{6,7}$

Interest in POCT is increasing because of technological advances that enable smaller and simpler

This article was externally peer reviewed.

Submitted 6 August 2015; revised 15 December 2015; accepted 4 January 2016.

From the Department of Family Medicine, University of Illinois at Chicago.

Funding: none.

Conflict of interest: none declared.

Corresponding author: Augustine J. Sohn, MD, MPH, Department of Family Medicine, University of Illinois at Chicago, 1919 W. Taylor St, M/C 663, Chicago, IL 60612 (E-mail: ajsohn@uic.edu). analytic devices that can provide measurements to diagnose and monitor conditions at the bedside and in physician offices at lower costs. Furthermore, POCT can improve patients' satisfaction with chronic disease management, especially in cases of type 2 diabetes mellitus and dyslipidemia, when results are immediately available and provide "teachable moments" during patient-physician encounters. ${ }^{8}$

Because of increasing interest among physicians and patients in implementing POCT in physician offices, and because manufacturers of point-of-care tests need to know what tests are valued by physicians, an international survey of primary care physicians was conducted in 5 countries. ${ }^{9}$ This report provides a more in depth report of US physicians' current and desired future POCT use and their opinions about the impact of health care reform on POCT.

\section{Methods}

This report uses the US data from a cross-sectional survey of primary care physicians in Australia, Belgium, the Netherlands, the United Kingdom, and the United States. A detailed description of the 
study methods of the international study, including survey development, is available elsewhere. ${ }^{9}$

\section{Survey}

The survey asked primary care physicians to identify up to 5 health conditions for which POCT might be helpful in making diagnoses, regardless of whether the tests were currently available. Respondents were also asked to identify up to 5 health conditions for which POCT might be helpful in monitoring or managing the condition, and up to 5 health conditions for which POCT might be helpful in reducing referrals. For each of these questions, respondents had the option to indicate that they did not believe POCT would be helpful. Respondents were then presented with the list of point-of-care tests and asked to indicate whether each test was currently available in their office and if they use it. For tests that respondents indicated were not currently available to them, physicians were asked to indicate whether they would use them, indicating desired use. Respondents indicating current or desired use were then asked to indicate how frequently they currently use or would use the test. Participants also responded to questions about the possible impact of health care or health care policy changes on POCT use.

The survey also collected information on the location of physicians' practices (urban, rural or suburban), years of practice, as well as their age and sex.

\section{Participants and Data Collection}

The target sample size for US physicians was 383 . This would allow relatively narrow confidence intervals for the point estimates for POCT use.

Data were collected in 3 phases. The survey request was initially sent to a random selection of 2000 active American Academy Family Practice members as well as 552 members of the American Academy Family Practice National Research Network. A total of 101 family physicians completed the survey in September 2013. Because of the low response, MDLinx, a commercial physician polling service, was contacted. The survey request was sent to 3000 regionally representative family physicians who are members of the MDLinx panel, and 298 complete surveys were received from these physicians, each of whom received $\$ 20$ for completing the survey. State family physician research networks in Oklahoma and Oregon were contacted as
Table 1. Top 10 Conditions for Which a Point-of Care Test Could Help Make a Diagnosis

\begin{tabular}{|c|c|c|}
\hline \multirow[b]{2}{*}{ Condition } & \multicolumn{2}{|c|}{$\begin{array}{l}\text { Respondents } \\
(\mathrm{N}=405)\end{array}$} \\
\hline & No. & $\%$ \\
\hline Diabetes mellitus & 229 & 57 \\
\hline UTI & 225 & 56 \\
\hline Strep throat & 218 & 54 \\
\hline Influenza & 175 & 43 \\
\hline Pregnancy & 103 & 25 \\
\hline Anemia & 72 & 18 \\
\hline Infectious mononucleosis & 60 & 15 \\
\hline INR/anticoagulation & 57 & 14 \\
\hline Acute cardiac condition & 55 & 14 \\
\hline Lipid disorder & 48 & 12 \\
\hline
\end{tabular}

INR, international normalized ratio; UTI, urinary tract infection.

well, and 6 of these physicians completed the survey for a total of 405 participants.

\section{Statistical Analyses}

Data were analyzed using SPSS (version 22; IBM/ SPSS, Inc., Chicago, IL). The International Classification of Primary Care was used to categorize the responses to open-ended questions about conditions for which physicians would like to use POCT. Coding was performed by 1 person (FA), and any ambiguities were resolved by a physician coauthor (AJS). Descriptive statistics were used to display the frequency for each condition. They were also generated for tests currently used or desired by all respondents, as well as frequency of tests. Responses to open-ended questions about changes in health care policy were coded by the main author (AJS).

\section{Results}

A total of 405 physicians responded from among the 5553 physicians who were sent the E-mail invitation, giving a response rate of $7 \%$. Of respondents, $71 \%$ were male and $29 \%$ were female; $47 \%$ practiced in an urban location, $25.2 \%$ in a suburban location, $21.2 \%$ in a semirural location, and $5.9 \%$ in a rural location.

Table 1 displays the top 10 conditions for which respondents indicated that POCT could help make a diagnosis. Diabetes mellitus was the most frequently cited condition, followed by urinary tract infection and strep throat. 
Table 2. Top 10 Conditions for Which a Point-of Care Test Could Help Monitor or Manage

\begin{tabular}{|c|c|c|}
\hline \multirow[b]{2}{*}{ Condition } & \multicolumn{2}{|c|}{$\begin{array}{c}\text { Respondents* } \\
(\mathrm{N}=110)\end{array}$} \\
\hline & No. & $\%$ \\
\hline Diabetes mellitus & 99 & 90 \\
\hline INR/anticoagulation & 61 & 55 \\
\hline Lipid disorder & 37 & 34 \\
\hline Anemia & 33 & 30 \\
\hline Kidney disease & 27 & 25 \\
\hline COPD/asthma ${ }^{\dagger}$ & 20 & 18 \\
\hline Hypertension & 16 & 15 \\
\hline Hyper-/hypothyroidism & 16 & 15 \\
\hline UTI & 15 & 14 \\
\hline Heart failure & 11 & 10 \\
\hline
\end{tabular}

*A total of 113 respondents completed question 2; 3 reported that they did not believe that point-of-care tests could help them monitor conditions. However, the percentages are based on $\mathrm{N}=110$.

${ }^{\dagger}$ Spirometry.

COPD, chronic obstructive pulmonary disease; INR, international normalized ratio; UTI, urinary tract infection.

Table 2 displays the top 10 conditions for which respondents indicated that POCT could help to monitor or manage the condition. Again, diabetes mellitus was the most frequently listed condition, followed by anticoagulation, lipid disorder, and anemia.

Table 3 displays the top 10 conditions for which respondents indicated that POCT would help to reduce referrals for specialty care or hospital admission. Acute cardiac condition, heart failure, and pulmonary embolism were the top 3 most frequently listed conditions.

Figure 1 displays point-of-care tests in descending order of use or potential use. The percentages indicate the proportion of respondents who use or would use each point-of-care test $\geq 1$ time/week, among those reporting current or desired use for each test. More than $80 \%$ of the physicians who responded to the survey indicated current or desired use of a throat swab for the group A streptococci test, a urine pregnancy test, blood glucose and $\mathrm{HbA}_{1 \mathrm{c}}$ tests, urine leukocyte or nitrite test, a nose/ throat swab for influenza, fecal occult blood test, INR test, hemoglobin test, chlamydia test, blood white cell count, and gonorrhea test. The frequency of current or desired use was also high for these tests: between $72 \%$ and $96 \%$ of those who use or would use these tests reported that they use or would use them $\geq 1$ time/week.

All 405 primary care physicians responded to the question, "Do you think current changes in US health care or policy are likely to have any impact on the use of POCTs? If so, please explain." Of the respondents, $63 \%(n=258)$ expressed the belief that POCT use will be affected by current changes in health care or policy; $23 \%(n=94)$ believed that POCT use would not be affected, and 14\% ( $\mathrm{n}=$ 56) were unsure.

Among those who believed that health care or policy changes would affect POCT use $(\mathrm{n}=258)$, $36 \%(n=92)$ expect that POCT usage would increase. Select responses to this question are presented in Table 4. Of those who believed that health care or policy changes would affect POCT use, $44 \%(n=114)$ expected POCT usage to decrease. Twenty percent (52 of 258), expect health care or policy changes to have a mixed effect on POCT use.

A total of 317 physicians responded to the survey item, "Please share any other comments, including benefits and concerns about POCT." Of these physician, 120 (38\%) identified benefits, 87 (27\%) identified concerns, and 17 (5\%) identified both

Table 3. Conditions for Which a Point-of Care Test Would Help Reduce Referrals for Specialty Care or Hospital Admission

\begin{tabular}{|c|c|c|}
\hline \multirow[b]{2}{*}{ Condition } & \multicolumn{2}{|c|}{$\begin{array}{l}\text { Respondents } \\
(\mathrm{N}=100)\end{array}$} \\
\hline & No. & $\%$ \\
\hline Acute cardiac condition & 39 & 39 \\
\hline Heart failure & 31 & 31 \\
\hline Pulmonary embolism & 26 & 26 \\
\hline Diabetes mellitus & 24 & 24 \\
\hline Anemia & 17 & 17 \\
\hline UTI & 16 & 16 \\
\hline COPD/asthma* & 15 & 15 \\
\hline Acute bronchitis & 14 & 14 \\
\hline Kidney disease & 13 & 13 \\
\hline INR/anticoagulation & 10 & 3 \\
\hline
\end{tabular}

*A total of 113 respondents completed question 3; 13 reported that they did not believe that point-of-care tests could help them reduce referrals. However, the percentages are based on $\mathrm{N}=$ 100.

${ }^{+}$Spirometry.

COPD, chronic obstructive pulmonary disease; INR, international normalized ratio; UTI, urinary tract infection. 
Figure 1. The numbers of respondents who use or would use the point-of-care testing $\geq 1$ time a week (black bar) among those reporting current or desired use (full bar). The percentages indicate the proportion of respondents. ALT, alanine aminotransferase; ANA, antinuclear antibody; AST, aspartate aminotransferase; BNP, brain natriuretic peptide; CRP, C-reactive protein; ESR, erythrocyte sedimentation rate; GGT, gamma-glutamyl; hCG, human chorionic gonadotropin; HDL, high-density lipoprotein; INR, international normalized ratio; LDL, low-density lipoprotein; MRSA, methicillin-resistant Staphylococcus aureus; PSA, prostate-specific antigen; TSH, thyrotropin-releasing hormone.

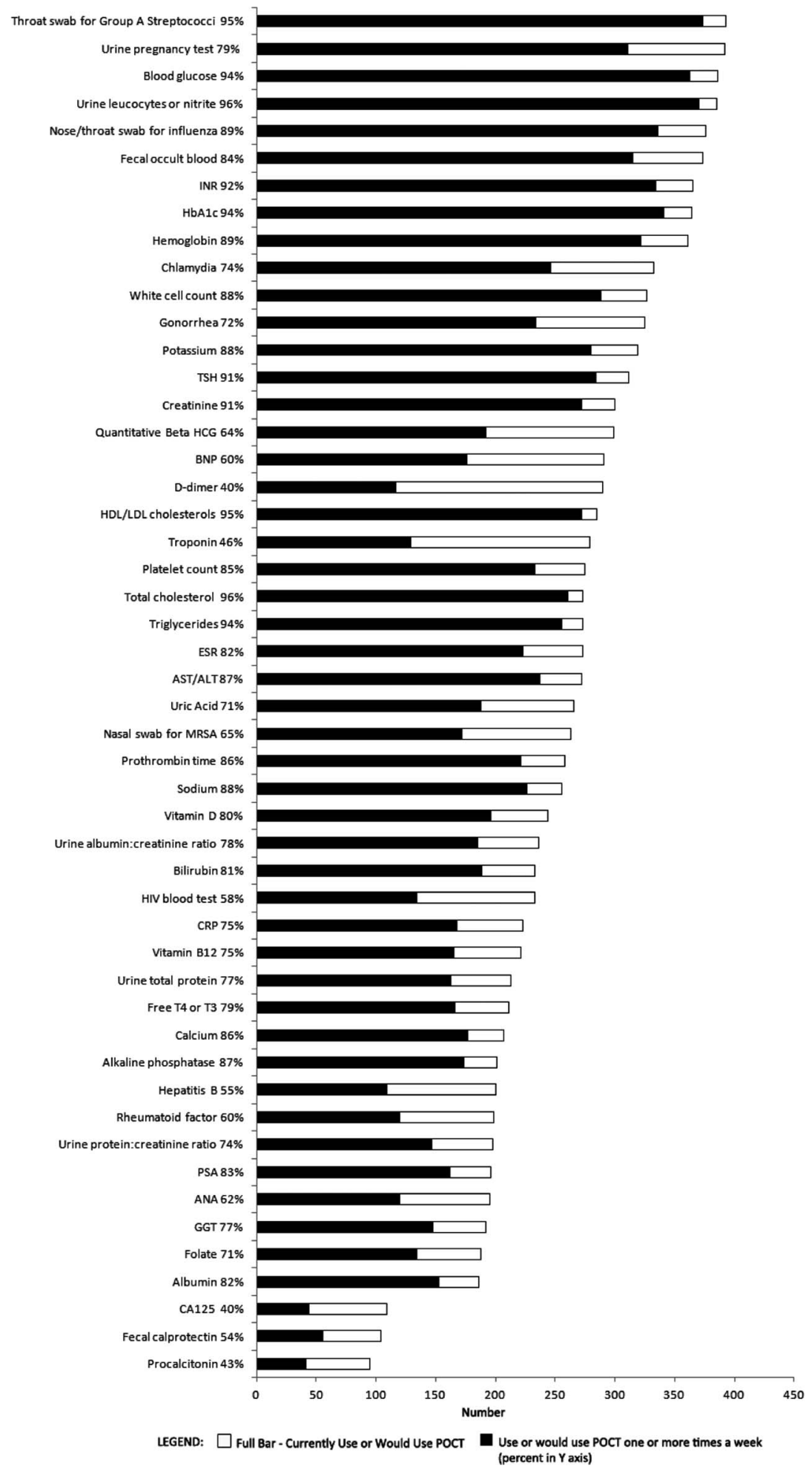


Table 4. Sample Comments about the Potential Impact of Health Care Policy Changes on the Use of Point-of-Care Testing

\begin{tabular}{l}
\hline Response Group \\
$\begin{array}{l}\text { Believe that policy changes will increase POCT } \\
\text { use }\end{array} \begin{array}{r}\text { "Yes, if there are recommendations to use these tests by established, evidence- } \\
\text { based guidelines, they will be used." } \\
\text { "Yes, as family medicine becomes more important in the health care arena, we } \\
\text { will need more tests to care for the total person and to refer appropriately." } \\
\text { "Yes, with patient-centered medical home." } \\
\text { "Yes, with more patients eligible for primary care, there will be more } \\
\text { problems to diagnose and POCT will be an important tool for the primary } \\
\text { care doctors to care for patients efficiently and at lower cost." } \\
\text { "I believe that the government will severely limit what can be done via } \\
\text { POCT." } \\
\text { "Yes. Less reimbursement or approval. Need for prior authorization." } \\
\text { "I think POCT will be more limited due to cost." } \\
\text { "Yes, I think that CMS will begin to regulate quality control of these tests." } \\
\text { "Yes. We need to be paid for doing the tests to make it worth the time and } \\
\text { training hassle. The amount needs to reflect the added patient convenience } \\
\text { of not having to do the lab." }\end{array}$ \\
$\begin{array}{c}\text { Believe that policy changes will decrease } \\
\text { Believe that policy changes will have a mixed } \\
\text { effect on POCT use }\end{array}$ \\
\hline
\end{tabular}

CMS, Centers for Medicare and Medicaid Services; POCT, point-of-care testing.

benefits and concerns. Table 5 provides some sample responses to this question.

\section{Discussion}

POCT has traditionally been used to diagnose acute conditions in outpatient care settings and to diagnose pregnancy. All the top conditions for which POCT could help make a diagnosis, as iden- tified by physicians in this study, are acute conditions (except diabetes mellitus). Our study shows that diabetes mellitus is the most frequent condition for which primary care physicians currently report making a diagnosis using POCT; this aligns with the introduction of hemoglobin $\mathrm{A}_{1 \mathrm{c}}\left(\mathrm{HbA}_{1 \mathrm{c}}\right)$ as a tool for the diagnosis of diabetes mellitus. With the increasing epidemic of obesity and type 2 dia-

Table 5. Sample Comments about Benefits of and Concerns about Point-of-Care Testing

\begin{tabular}{l}
\hline Response Group \\
\hline Benefits of POCTs \\
"Quick answers allow immediate feedback to patients." \\
"POCTs are vital to appropriate diagnosis and appropriate management of many commonly \\
treated conditions." \\
"I have had in house lab for years, and it has saved a few lives or reduced significant morbidity. \\
POCT are [sic] the Future." \\
"Judicious use of POCTs in the future means overall healthcare savings." \\
"I believe changing medication doses at the time of visit is much more impactful instead of \\
calling the patient later. Quick diagnosis of UTI, strep throat etc. also allows for appropriate \\
quick treatment." \\
"Being able to monitor quality/run controls" \\
"Concern of accuracy of some of these tests—false negatives and positives." \\
"Payment in a CLIA waived office is really an issue." \\
"Reimbursement [from] insurance inhibits risk to acquire equipment and maintenance needs." \\
"Only concerns would reimbursement from insurance, the initial investment cost, and regulation \\
usage \\
"Trom the government." \\
"They can be quite useful for patient feedback, but if they are not reliable, they are less helpful." \\
"Concerns over sensitivity and specificity of test results, helpful to be able to diagnose infections \\
earlier, helpful to monitor critical values such as the INR more quickly." \\
"Benefit—fast result, concerns—false negative."
\end{tabular}

CLIA, Clinical Laboratory Improvement Amendments; INR, international normalized ratio; POCT, point-of-care testing; UTI, urinary tract infection. 
betes mellitus-not only in the United States, but also in Asia and Oceania-the availability of POCT for $\mathrm{HbA}_{1 \mathrm{c}}$, in addition to blood glucose, is very helpful in diagnosing new-onset diabetes mellitus and in monitoring treatment response. Fasting is not required for $\mathrm{HbA}_{1 \mathrm{c}}$, which is an advantage over using the fasting blood glucose measure. Now that testing of lipid concentrations does not require fasting ${ }^{10}$ (except for patients with markedly elevated triglycerides), the 3 major coronary artery disease risk factors that can be controlled with treatment (glucose, lipids, blood pressure), can be monitored during office visits, both giving immediate feedback to patients and allowing immediate treatment changes to be made.

To our knowledge, this is the first US study to elicit primary care physicians' opinions regarding the use of and desire for POCT among a national sample of physicians, detailing the usefulness of these tests for diagnosis and monitoring and for reducing referrals. A limitation of this study is that the survey measured physicians' self-reported use and opinions of POCT, rather than objectively measuring their behavior. Whether the referral rates would actually decrease, for example, could only be determined by measuring actual referrals, preferably in a randomized trial. A second limitation is the low response rate, though the written comments enrich the survey results. The response rate for the same survey among family physicians in the 4 other countries where the survey was administered varied from $10 \%$ to $68 \%$. ${ }^{9}$ Responses regarding the use of point-of-care urine pregnancy, urine leukocyte or nitrite, and blood glucose tests were similar among family physicians from the United States and the other countries. However, US physicians tend to use more point-of-care fecal occult blood tests, throat swabs for group A streptococci, nasal swabs for influenza, and $\mathrm{HbA}_{1 \mathrm{c}}$ tests.

The attitude of US physicians toward the anticipated impact of US health policy change on POCT is mixed. More physicians predicted decreased use than increased use. This may reflect the overall pessimistic attitude of physicians about the health policy changes implemented by the Affordable Care Act and its implications and meaningful use requirements, which have been burdensome for many primary care physicians. Nonetheless, US physicians cited more benefits than concerns about POCT use; $43 \%$ mentioned benefits as opposed to $32 \%$ who mentioned concerns. The cited benefits agree with prior studies that demonstrated decreased turnaround time for results leading to increased patient and physician satisfaction.

\section{Conclusion}

Many physicians reported they use or would use POCT now and in the future, suggesting a real opportunity for continued expansion of POCT technology. If point-ofcare tests are highly reliable, easy to use, and inexpensive, it is highly likely their use will increase dramatically, leading to better diagnosis of acute and chronic medical conditions, better management of chronic medical conditions such as diabetes mellitus, and improved physician and patient satisfaction. POCT may also decrease referrals for specialty care and to emergency departments, but this hypothesis needs to be tested.

\section{References}

1. Price C, St. John A. Point-of-care testing. 2nd Ed. Washington, DC: AACC Press; 2004.

2. Miller C, Barnes C, Philips L, et al. Rapid A1c availability improves clinical decision-making in an urban primary care clinic. Diabetes Care 2003;26: $1158-63$.

3. Kennedy L, Herman W, Strange P, et al. Impact of active versus usual algorithmic titration of basal insulin and point-of-care versus laboratory measurement of HbA1c on glycemic control in patients with type 2 diabetes. Diabetes Care 2006;29:1-8.

4. Al-Ansary L, Farmer A, Hirst J, et al. Point-of-care testing for $\mathrm{HbAlc}$ in the management of diabetes: a systemic review and analysis. Clin Chem 2011;57:568-76.

5. Bagley A, Malabu U. Diabetes epidemic in the Asia Pacific region: has hemoglobin A1c finally earned its place as a diagnostic tool? Asian Pac J Trop Biomed 2014;4:85-9.

6. Bubner T, Laurence C, Gialamas A, et al. Effectiveness of point-of-care testing for therapeutic control of chronic conditions: result from the PoCT in general practice trial. Med J Aust 2009;190:624-6.

7. Gialamas A, St. John A, Laurence C, et al. Point-ofcare testing for patients with diabetes, hyperlipidemia or coagulation disorders in the general practice setting: a systemic review. Fam Pract 2010;27:17-24.

8. Crocker B, Lewandrowski E, Lewandrowski N, et al. Patient satisfaction with point-of-care laboratory testing: report of a quality improvement program in an ambulatory practice of an academic medical center. Clin Chim Acta 2013;424:8-11.

9. Howick J, Cals J, Jones C, et al. Current and future use of point-of-care tests in primary care: an international survey in Australia, Belgium, the Netherlands, the UK and the USA. BMJ Open 2014;4:e005611.

10. Doran B, Guo Y, Xu J, et al. Prognostic value of fasting versus nonfasting low-density lipoprotein cholesterol levels on long-term mortality: insight from the National Health and Nutrition Examination Survey III (NHANES-III). Circulation 2014; 130:546-53. 